\title{
La educación médica en Rusia
}

\author{
F. Vartanian
}

\section{Introducción}

La educación médica en Rusia se remonta al siglo $\mathrm{XI}$; sin embargo la enseñanza reglada de medicina se inició con la segunda fundación de la Universidad de Moscú en 1755 y con la creación de su Facultad de Medicina en 1764. La formación médica primigenia fue similar a la adoptada en Alemania y los Países Bajos. A comienzos del siglo xx, en Rusia existían 16 Facultades de Medicina, mientras que en 1935 ya eran 55 .

En 1930, las Facultades de Medicina fueron separadas de las universidades y pasaron a denominarse Institutos Médicos, adquiriendo un estatus independiente bajo la supervisión del Ministerio de Sanidad. Esta medida se tomó para que la educación médica se aproximara a los organismos gubernamentales de perfil similar y para conseguir su mejor integración con los servicios sanitarios. También se establecieron sistemas de Institutos Médicos para los niveles formativos básico y posgraduado.

\section{Educación médica básica}

La educación médica básica requiere seis años de formación que comienza generalmente a los 17 años. La formación de médicos y farmacéuticos en Rusia actualmente se realiza en 48 instituciones de educación superior.

La educación médica es principalmente subsidiada por el Estado, que planifica según las necesidades nacionales de personal médico. $\mathrm{La}$ financiación de la educación superior en medici- na proviene de los presupuestos estatales. El Ministerio de Sanidad coordina el trabajo educativo, metodológico y organizativo de las redes institucionales y fija el número de alumnos admitidos.

La función planificadora y de gestión a todos los niveles asegura el funcionamiento del sistema y su relevancia para los servicios asistenciales y las necesidades educativas de la profesión médica de forma continuada. En Rusia, las organizaciones responsables del desarrollo y revisión de los estándares profesionales también son las encargadas de diseñar los programas formativos de las escuelas de medicina; son responsables de la producción de los recursos necesarios para el sistema de salud nacional y del desarrollo de los estándares profesionales y de la calidad de las especialidades para las instituciones educativas de formación especializada y para la formación médica continuada (FMC) obligatoria para la recertificación periódica. La evaluación regular de las habilidades, el conocimiento, las actitudes y las capacidades para definir problemas es otra función compleja que se lleva a cabo de forma continuada a los diferentes niveles y en intervalos especificados por distintas instituciones, Todo ello se lleva a cabo en el contexto legal según los requisitos establecidos para las diferentes dimensiones de FMC.

Las instituciones están abiertas a los solicitantes que han completado una formación secundaria o la escuela de enfermería. Los contenidos de los exámenes de admisión tratan cuestiones de biología, física, química e idiomas. La admisión en los institutos médicos es un proceso altamente competitivo.

Las instituciones médicas superiores se organizan en cinco facultades: la de Medicina Curativa, cuyo currículo es de seis cursos anuales y gradúa médicos
Vicerrector de la Academia Rusa de Estudios Médicos Avanzados. Moscú, Rusia.

Correspondencia Profesor Felix Vartanian. Academia Rusa de Estudios Médicos Avanzados.

Barricadnaya, 2

Moscú, Rusia.

E-mail felcart@gmail.com 


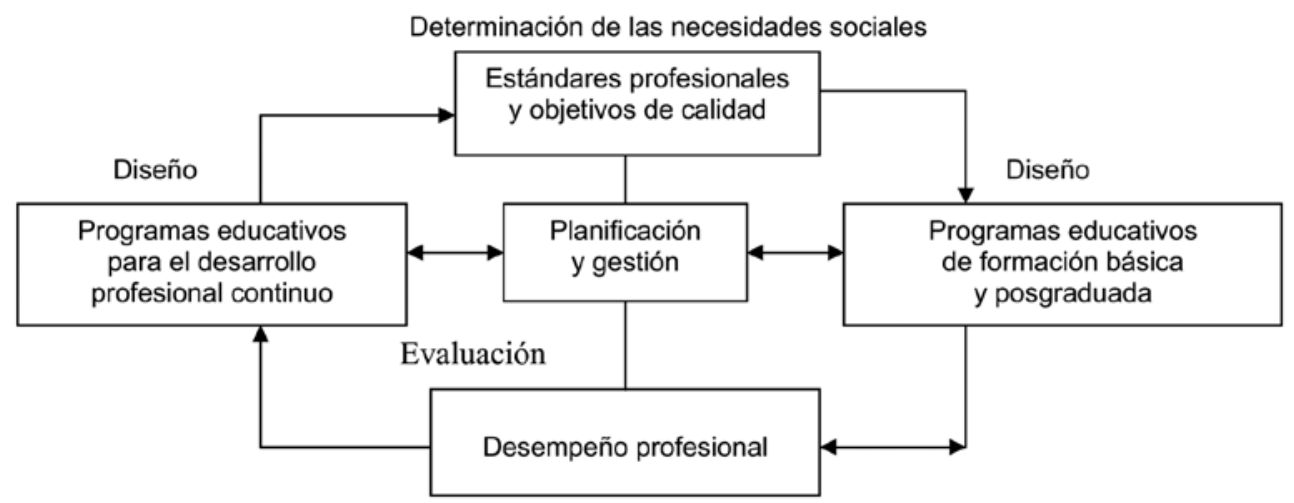

Figura 1. Modelo conceptual de la educación médica.

generales; la de Pediatría, con seis años de estudio y que produce especialistas pediatras; la de Higiene, también con un currículo de seis años, que produce especialistas en higiene sanitaria; la de Estomatología, carrera de cinco años que produce estomatólogos, y la de Farmacia, con un currículo de cuatro años que produce especialistas farmacéuticos.

El contenido del currículo es el mismo para todas las facultades en los dos primeros años e incluye las ciencias médicas preclínicas, que son fundamentales como base para cualquier especialidad médica. La especialización médica comienza en el tercer curso, e incluye propedéutica, bioquímica y patología clínica (anatomía y fisiología patológica), así como materias especiales que varían según la orientación generalista o pediátrica. La característica principal del currículo, tanto de medicina general como de pediatría, es un nuevo enfoque formativo que incorpora dos años de especialización en atención primaria, uno antes y otro después de la graduación.

El currículo en estas facultades está diseñado para cubrir la totalidad de los contenidos de la educación médica general en cinco años. Durante los cursos tercero, cuarto y quinto, los estudiantes despliegan actividades como enfermeros, feldshers y asistentes clínicos. En el sexto año, los estudiantes de medicina toman su especialización primordial en uno de los ámbitos clínicos siguientes: cirugía, obstetricia y ginecología en la Facultad de Medicina Curativa; pediatría, que incluye las enfermedades infecciosas de la infancia, y cirugía pediátrica, que incluye ortopedia. La subespecialización clínica no es posible durante la formación básica debido a que la adquisición de un conocimiento profundo de las materias clínicas primordiales es fundamental para la formación adecuada de los médicos

Después del sexto año, los estudiantes deben superar el examen de estado correspondiente a su titulación para obtener el diploma de Doctor y completar un internado de un año en una unidad asistencial trabajando bajo la supervisión de especialistas. La instrucción en oftalmología, otolaringología, dietética, climatoterapia, endocrinología, bioquímica clínica, fisioterapia y algunos aspectos de cirugía, como la oncología y la neurocirugía, corre a cargo de los departamentos correspondientes.

La metodología educativa en las instituciones médicas de educación superior incluye lecciones magistrales dictadas por los mejores especialistas y clases teóricas regulares a lo largo de todo el periodo formativo.

Los programas formativos se ajustan a las necesidades poblacionales de acuerdo a las prioridades en salud. Así, cuando en los años cincuenta del siglo pasado la enfermedades no contagiosas se convirtieron en un problema de salud, se reforzó su presencia en los contenidos curriculares, y cuando en los años setenta el papel de la atención primaria se hizo evidente, los programas formativos se adaptaron para cubrir este objetivo educativo.

Después de los exámenes finales de cuarto y quinto año, los estudiantes de todas las facultades utilizan los periodos de vacaciones académicas intersemestrales en formación profesional. Aprenden a utilizar así su conocimiento, a desarrollar sus habilidades profesionales, a familiarizarse con 


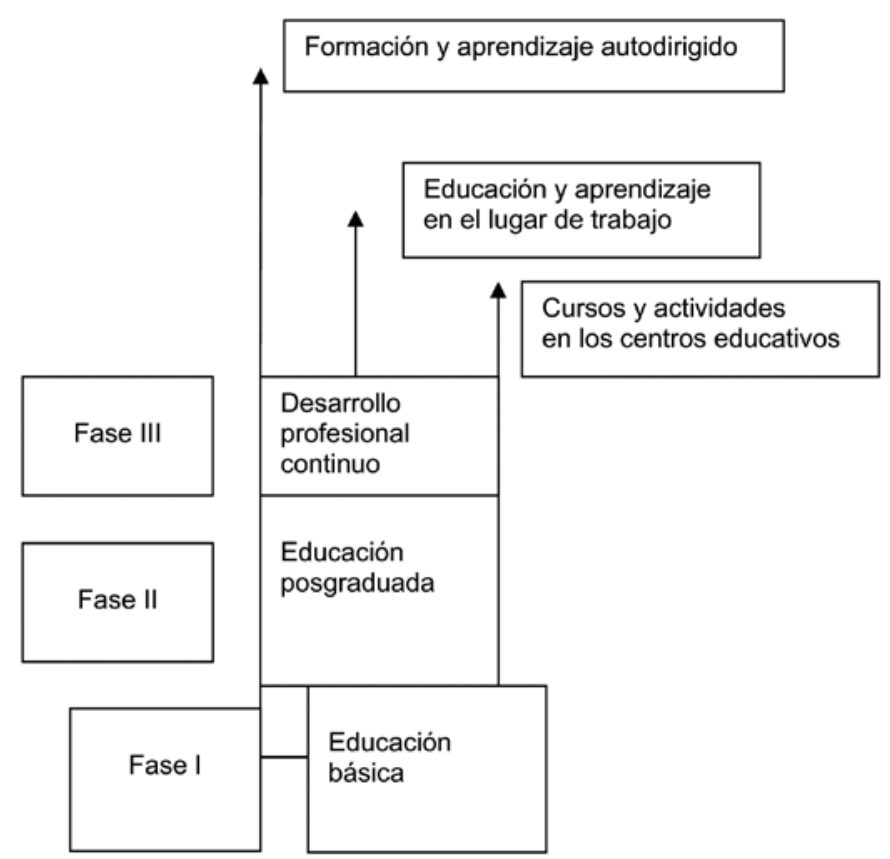

Figura 2. Educación médica continuada en el conjunto educativo.

los métodos actuales de diagnóstico y tratamiento y con las tareas que tendrán que desarrollar en el futuro. Por lo menos, el $25 \%$ del tiempo de formación de todas las especialidades se dedica a la práctica en atención primaria.

Finalmente, los estudiantes deben superar un examen de estado sobre los temas generales y los específicos para conseguir el diploma de médico de medicina general en las Facultades de Medicina Curativa, de médico pediatra en la Facultades de Pediatría, de oficial sanitario en las Facultades de Higiene, de médico estomatólogo en las Facultades de Estomatología, o de farmacéutico en las Facultades de Farmacia.

Cada año se gradúan unos 60.000 estudiantes de las instituciones de educación superior médica.

\section{Educación médica posgraduada}

La educación médica posgraduada y el desarrollo profesional continuado de los médicos en la Federación Rusa es comprehensiva y sistemática, quizás de forma única. La primera experiencia exitosa en establecer un prototipo sistémico de formación posgraduada fue desarrollada por el Instituto de Formación Médica Posgraduada de San Petesburgo un siglo atrás,

La implementación de los programas de posgrado y del desarrollo profesional son gestionados por el Ministerio de Salud a través de sus departamentos de educación médica y de los regionales. La mayor parte de la tarea es realizada por instituciones educativas estatales que incluyen 48 facultades para el desarrollo profesional continuo, adscritas a instituciones de formación básica, así como institutos independientes especializados en el desarrollo profesional continuo de los médicos.

En el ámbito nacional, 35.000 médicos especialistas cualificados asisten anualmente a los cursos de la Academia Rusa de Estudios Médicos Avanzados siguiendo una planificación de objetivos especificados por el Ministerio de Salud y Desarrollo Social -a partir del 2002 se integraron los programas de desarrollo social y de salud en el órgano gubernamental que pasó a denominarse Ministerio de Salud y Desarrollo Social-, en base a las necesidades de atención sanitaria. 
En el ámbito regional, una proporción aún mayor de personal de la salud se encuentra regularmente involucrado en la formación en hospitales regionales altamente especializados y otras instituciones proveedoras de servicios asistenciales. Además, en los distritos, miles de profesionales se encuentran simultáneamente involucrados en actividades formativas en su lugar de trabajo. Los programas formales educativos para las especialidades médicas son diseñados, revisados periódicamente y exigibles para los ocho Institutos de Desarrollo Profesional Medico Continuo. Los programas de formación regionales, de distrito o locales generalmente se desarrollan en sus propios niveles para garantizar su relevancia en relación a las necesidades y los problemas locales.

Las opciones en FMC son múltiples, pero existen tres patrones primordiales: autoaprendizaje en casa, formación en el lugar de trabajo y actividades formales en los institutos y facultades para el desarrollo profesional continuo. En el ámbito nacional, regional y local son miles los profesionales que siguen programas formales en instituciones educativas, mientras que son muchos más los que lo hacen en su lugar de trabajo tomando parte en seminarios, conferencias o ejercicios de equipo organizados. Todos los profesionales, además, practican el autoaprendizaje individual de forma permanente.

También existe el nivel de educación posgraduada, con un programa de naturaleza residencial y de dos años de duración ('ordinatura') diseñado para la formación clínica y, en algunos casos, la capacitación supervisora necesaria en departamentos y hospitales, que dependen del nivel académico universitario.

La motivación es un factor clave, por lo que es necesario tener en cuenta que la promoción (carrera profesional) depende claramente de haber completado con éxito una formación avanzada, siendo un prerrequisito para alcanzar las categorías superiores, con salarios sustancialmente más elevados. Se concede a cada médico un certificado estándar al graduarse de cada curso, en tanto que las fechas y la duración de éste se registran en la ficha personal de cada médico.

Cada vez se intensifica más el diseño de actividades sistematizadas y todas las formas de 'aprender haciendo', en tanto que las lecciones magistrales se van reduciendo paulatinamente, no sin resistencias, en beneficio del aprendizaje basado en la 'solución de problemas' y en la 'toma de decisiones. Este tipo de aprendizaje se ha mostrado más motivador y efectivo que la didáctica tradicional. Aunque la importancia del desarrollo profesional continuo es ampliamente reconocida, sólo algunos pocos creen saber cómo se miden sus resultados positivos o los beneficios directos y tangibles de dicha formación de los profesionales de la salud.

Anualmente, unos 150.000 especialistas siguen los distintos cursos de los programas de desarrollo profesional continuo ofrecidos por las instituciones educativas. Los cursos que se ofrecen cubren prácticamente el espectro completo de la medicina actual.

\section{Colaboración internacional en educación médica}

La historia de la colaboración internacional global y regional es muy rica e interesante. La cooperación internacional ha estimulado desarrollos positivos de la educación médica durante largo tiempo. Organizaciones intergubernamentales y no gubernamentales han intensificado dicha colaboración en las últimas tres décadas.

La Organización Mundial de la Salud (OMS), la Federación Mundial de Educación Médica (WFME) y otras organizaciones internacionales avanzaron en la creación de contactos en el ámbito mundial organizando proyectos conjuntos de investigación y programas formativos, conferencias, congresos y reuniones. El espectro profesional de todas estas actividades ha sido muy amplio, abarcando desde las disciplinas clínicas hasta cuestiones de salud pública. Todo ello ocurría en el siglo pasado. Sin embargo, en la última década se observa una disminución de las actividades de las organizaciones internacionales que actúan en el campo de la educación médica y del desarrollo de los recursos humanos de la salud.

La utilidad de la colaboración internacional benefició a muchos países. Los resultados de las actividades de la OMS en el pasado se incorporaron a los currículos de medicina y a los programas de formación de especialistas y del desarrollo profesional continuo. Los temas principales de la Declaración de Edimburgo (1988) fueron de gran utilidad para el desarrollo de la formación médica. Múltiples instituciones educativas establecieron colaboraciones multilaterales en el extranjero que perduran al tenerse en gran estima; por ejemplo, nuestra Academia ha mantenido durante muchos 
años una estrecha colaboración con el Institut d'Estudis de la Salut de Cataluña. Las actividades colaborativas con participación de la OMS fueron de gran utilidad desde la perspectiva profesional. Actualmente, la Academia Rusa colabora con el proyecto internacional GLOBE, dedicado a la promoción de la atención primaria a través de la formación del personal sanitario que trabaja en este importante sector sanitario. Considerando la citación mundial actual y sus tendencias migratorias, entre las cuales no pueden ignorarse las de especialistas médicos, es evidente la necesidad de promover internacionalmente procesos de acreditación profesional reconocidos a través de las fronteras. El intercambio de información de los programas de formación y de los requisitos para la certificación de la licencia de ejercicio profesional facilita, sin duda, la predisposición para ello. Esta tarea, además, puede ser muy útil para promocionar las mejoras en la educación médica dentro de los propios países. Estos esfuerzos combinados deben relacionarse con la calidad asistencial, que es actualmente una prioridad en salud. De nuevo, los problemas de salud pública devienen vitales en todas partes. La cooperación necesaria en este campo aporta nuevas promesas e ideas para el desarrollo de la salud. La situación sanitaria en la Federación Rusa y las ideas acerca de las reformas sociales, incluyendo el sector de la salud, requieren nuevos modelos optimizados de la atención y una filosofía de salud pública innovadora. Finalmente, la colaboración internacional, en general, y los recursos humanos de la salud, en particular, deben centrarse en un desarrollo a lo largo del siglo XxI, durante el cual es posible soñar en un mundo unido, por lo menos en lo que respecta a la medicina.

\section{Conclusión}

La educación médica y su desarrollo está al servicio de los sistemas de salud y de las necesidades de la población. Por tanto, la orientación educativa dependerá de la situación de salud poblacional en cada país. Si somos capaces de analizar y predecir el desarrollo requerido por el sistema sanitario, veremos también las perspectivas evolutivas de la educación médica.
Es esencial subrayar el papel de las autoridades centrales en la monitorización y orientación de los cambios educativos en los niveles básico, posgraduado y de desarrollo profesional continuo, para asegurar que los médicos puedan proveer servicios de alta calidad.

Analizando la educación médica y su historia se llega fácilmente a la conclusión de que se trata de un fenómeno de los más estables de entre todas las actividades humanas.

Así, durante los últimos veinte años se han producido evidentes cambios políticos y económicos en el seno de la antigua Unión Soviética. Sin embargo, cuando se compara la situación actual de la educación médica en Rusia con el sistema educativo soviético se encuentran muy pocas diferencias, tan sólo localizadas en la denominada 'educación de pago', que atiende a estudiantes y especialistas que eran excluidos en los tiempos del poder soviético.

El futuro del sistema de salud, de la investigación biomédica y de la educación médica están inseparablemente unidos a la situación económica del país. Si, por ejemplo, no llegara una suma adicional sustancial de fondos para mejorar el estado actual del sistema asistencial gestionado por el Ministerio de Salud y Desarrollo Social, los cambios, por muy innovadores que fueran en la financiación y en la reorganización del uso de los recursos existentes actualmente, no mejorarían de manera significativa la calidad del sistema de salud, incluyendo la educación médica.

Esto lleva a la conclusión de que lo que tenemos hoy puede no ser aceptable en el futuro.

\section{Bibliografía}

1. Vartanian F. Continuing medical education in the Union of Soviet Socialist Republics. Philosophy and approaches. JAMA 1987; 258: 1358-60.

2. Vartanian F. Information technologies: their inevitable impact on CME (illusions, realities, perspectives). Journal of Continuing Medical Education in the Health Professions 1988; 8: 313-9,

3. Vartanian F. Continuing medical education and its management. J Manag Med 1993; 7: 29-34. 